\title{
Communication, transparency key as Canada faces new coronavirus threat
}

\author{
n Cite as: CMAJ 2020 February 18;192:E171-2. doi: 10.1503/cmaj.1095846
}

Posted on cmajnews.com on January 30, 2020

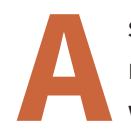

s Canada faces the threat of the new coronavirus spreading within its borders, there are important communication lessons to recall from the 2003 severe acute respiratory syndrome (SARS) outbreak, say infectious disease experts.

Canada recently confirmed its first case of the novel coronavirus that has infected thousands in China, spread to other countries, and led to the deaths of more than 100 people. There are now more confirmed cases of coronavirus infection in China than there were cases of SARS in 2003.

The public health landscape in Canada has changed dramatically since the SARS outbreak, says Dr. Ross Upshur of the Dalla Lana School of Public Health in Toronto. At the national level, "the machinery has been put in place for Canada to be well prepared," he says. Canada now has a national public health agency and a federal-provincial-territorial council to coordinate emergency public health response, as well as pan-Canadian agreements on information sharing and mutual aid. There are also "far more health professionals trained in public health and far more lab capacity," now than in 2003, according to Upshur.

However, he acknowledges that "much of what happens in an outbreak is outside of the control of that machinery." The success of the response to the new coronavirus also depends on the coordination and communications systems within hospitals. Upshur's research into recent pandemic responses showed that two-way communication between hospital decision makers and front-line providers is key. The same issues were highlighted in national reports in the wake of the SARS outbreak.

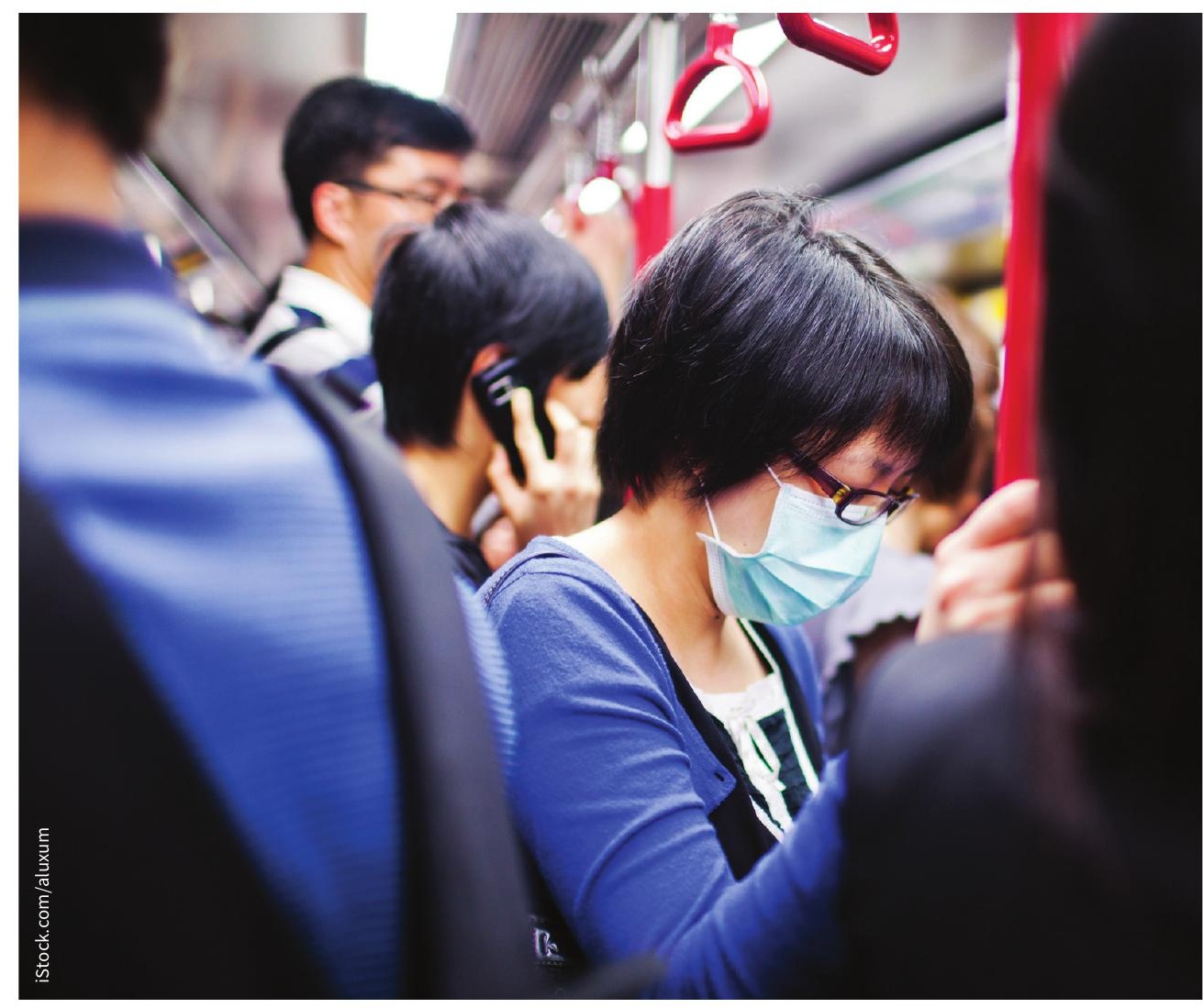

Public health preparedness has come a long way since the 2003 SARS outbreak, say health professionals.

"Trust is probably the most important glue that holds together epidemic response," says Upshur. "If health care professionals believe that they're not being told the complete truth, or that institutions aren't taking all steps necessary to ensure that they have all the resources required to carry out their work safely, they will push back." For example, they may not take directives seriously or may refuse work, he says.

It appears there may still be gaps in communication between public health and front line staff. Shortly after Toronto
Public Health officials announced the first case of the new coronavirus on Twitter, a union representing the paramedics who transported the patient raised concerns that the paramedics were not notified about the diagnosis. "Unfortunately, the paramedics who transported this patient just found this out from the media and your tweet," the union tweeted. Public health officials later told reporters they did not notify the paramedics because they were wearing protective garb.

According to Dr. Allison McGeer, director of infection control at Mount Sinai Hospital 
in Toronto, "one of the things that was hardest about SARS for many people was that the directives changed daily, because when you're in the middle of an outbreak of a novel disease, you're learning things every day." Without adequate explanation, "it's really hard to cope with the speed of that change," she says. "Should we have to deal with this again, it will be really important to get people to understand that yes, it's frustrating if [protocols] are changing, but it's good if they're changing."

According to Upshur, transparency is also vital to ensure the public cooperates in quarantine, reporting and surveillance measures. "We have really good role models from SARS," he says. Dr. Sheela Basrur, Toronto's chief medical officer of health at the time, and Dr. Don Low, then chief of microbiology at Mount Sinai, "were incredibly gifted risk communicators," Upshur says. "People were assured that they were being told the straight truth."
Dr. Isaac Bogoch, an infectious disease specialist at Toronto's University Health Network, is impressed so far with the public health communications about the new coronavirus. Within a couple of weeks of the first cases, he has seen "rapid communication about how samples should be acquired, how the samples should be processed, how to send the samples to the appropriate provincial health lab." By comparison, the SARS outbreak was marked by a relative lack of information flowing from officials both in Canada and China, where the virus originated.

Bogoch also praised the transparency shown by Canada's chief public health officer, Dr. Theresa Tam. "It's been very comforting to have Dr. Tam give several press conferences to the general public ... saying here's what we know. And she's also very comfortable saying here's what we don't know, but here's how we're going to prepare for the unknown."
Early estimates suggest that the virus is moderately contagious and the case fatality rate is about $3 \%$. But the number of confirmed cases is rising and it's not clear how Canada's already overcrowded hospitals would accommodate a surge in cases. According to McGeer, integrated health authorities in provinces like Alberta and British Columbia may have a leg up on less centralized systems. "That could be a structure that makes it much easier and more efficient to both communicate and run health care emergencies."

Federal public health officials have struck a special advisory committee with representatives from each province and territory to coordinate the response across Canada. Ontario's Chief Medical Officer of Health Dr. David Williams said the committee is meeting regularly as the situation evolves.

Wendy Glauser, Toronto, Ont. 